\title{
Use of virtual slide system for quick frozen intra-operative telepathology diagnosis in Kyoto, Japan
}

\author{
Yasunari Tsuchihashi*1, Terumasa Takamatsu'2 ${ }^{2}$ Yukimasa Hashimoto ${ }^{3}$, \\ Tooru Takashima*3, Kooji Nakano ${ }^{3}$ and Setsuya Fujita ${ }^{1}$
}

Address: ${ }^{1}$ Louis Pasteur Centre for Medical Research, 12-7 Taniguchi Kakinouchi, Ukyo, Kyoto, 616-8012, Japan, ${ }^{2}$ CLARO Inc., 56-10, Honcho, Hirosaki, Aomori, 036-8203, Japan and 'Yamashiro Public Hospital, 74-1, Kizu-Ikeda, Kizu, Kyoto, Japan

Email: Yasunari Tsuchihashi* - tsuchi@louis-pasteur.or.jp; Terumasa Takamatsu - terma@claro-inc.jp

* Corresponding authors

from 9th European Congress on Telepathology and 3rd International Congress on Virtual Microscopy

Toledo, Spain. 15-17 May 2008

Published: 15 July 2008

Diagnostic Pathology 2008, 3(Suppl I):S6 doi:10.1186/1746-1596-3-SI-S6

This article is available from: http://www.diagnosticpathology.org/content/3/SI/S6

(C) 2008 Tsuchihashi et al; licensee BioMed Central Ltd.

This is an open access article distributed under the terms of the Creative Commons Attribution License (http://creativecommons.org/licenses/by/2.0),

which permits unrestricted use, distribution, and reproduction in any medium, provided the original work is properly cited.

\begin{abstract}
We started to use virtual slide (VS) and virtual microscopy (VM) systems for quick frozen intraoperative telepathology diagnosis in Kyoto, Japan. In the system we used a digital slide scanner, VASSALO by CLARO Inc., and a broadband optic fibre provided by NTT West Japan Inc. with the best effort capacity of 100 Mbps. The client is the pathology laboratory of Yamashiro Public hospital, one of the local centre hospitals located in the south of Kyoto Prefecture, where a fulltime pathologist is not present. The client is connected by VPN to the telepathology centre of our institute located in central Kyoto. As a result of the recent 15 test cases of VS telepathology diagnosis, including cases judging negative or positive surgical margins, we could estimate the usefulness of VS in intra-operative remote diagnosis. The time required for the frozen section VS file making was found to be around $10 \mathrm{~min}$ when we use $\times 10$ objective and if the maximal dimension of the frozen sample is less than $20 \mathrm{~mm}$. Good correct focus of VS images was attained in all cases and all the fields of each tissue specimen. Up to now the capacity of best effort B-band appears to be sufficient to attain diagnosis on time in intra-operation. Telepathology diagnosis was achieved within 5 minutes in most cases using VS viewer provided by CLARO Inc. The VS telepathology system was found to be superior to the conventional still image telepathology system using a robotic microscope since in the former we can observe much greater image information than in the latter in a certain limited time of intra-operation and in the much more efficient ways. In the near future VS telepathology will replace conventional still image telepathology with a robotic microscope even in quick frozen intra-operative diagnosis.
\end{abstract}

\section{Introduction}

The practical applications of virtual slide (VS) or virtual microscopy (VM) system have been confined to such fields as education, conferences or consultations, where we are allowed enough time to digitize whole a slide specimen and make up a VS before starting observation. How- 
ever through the recent rapid technical progresses of digital slide scanner for VS, the time required for making up a VS file is greatly shortened to the order of $10 \mathrm{~min}$ or so, so that we can now utilized VS and VM for quick frozen intra-operative telepathology diagnosis. For the last 15 years our telepathology project in Kyoto, Japan has been conducted using a static image telepathology system equipped with a remote controllable robotic microscope [1]. The system can now be called "conventional", in contrast to the "novel", VS telepathology system. In this paper we report our first trial of applying VS telepathology system to quick frozen intra-operative telepathology diagnosis. Merits of the use of VS system will be discussed.

\section{Materials and methods}

We used a highly elaborate digital slide scanner, VASSALO, by CLARO Inc., Aomori, Japan [2] in a client pathology laboratory of Yamashiro Public Hospital in the south of Kyoto, Japan, where fulltime pathologist is not present by a well-trained cytotechnician is present. The client terminal is connected to our telepathology diagnostic centre in central Kyoto by a broadband optic fibre provided commercially by NTT-Wes Inc., Japan that has a best-effort capacity of $100 \mathrm{Mbps}$. The broadband was used solely for image data transmission and the client terminal and our telepathology diagnostic centre formed a VPN, called a B-Flets group. The time required for VS making of each frozen section was measured. At the same time, the time and accuracy of the remote observation of the VS by a VS viewer provided by CLARO Inc. were checked and recorded. Usefulness of VS telepathology diagnosis is estimated in terms of time required, VS quality, and of accuracy of diagnosis. The VS telepathology diagnosis was compared to those by conventional telepathology system by making diagnoses using VS and conventional systems successively for a same frozen section slide specimen.

\section{Results}

In the last 6 months we conducted 15 test cases of quick frozen VS diagnosis. The time required for VS making was around $10 \mathrm{~min}$ if we use $\times 10$ objective and if the specimen measures less than $20 \mathrm{~mm}$ in the maximal dimension. VS making was successful for all the tissue section cases. i.e., good focus of VS images was achieved in all the frozen section cases and in all the tissue fields of each case. Telepathology observation and diagnosis by VS viewer were achieved within $5 \mathrm{~min}$ and all the telepathology diagnoses were correct. The best effort type B-band optic fibre maintained sufficient capacity to attain remote diagnosis on line and on time.

\section{Discussion}

Owing to the rapid and accurate digitalization of the whole of a glass slide specimen by VASSALO it was now clear that we can successfully use VS telepathology system even for quick frozen intra-operative telepathology diagnosis where time is quite limited for a technician and a remote pathologist. Telepathology diagnosis was easier and more comfortable in the novel VS telepathology system than in the conventional telepathology system since we can obtain desired images easier in VS system than in the conventional system. We can attain much greater image information processing in the VS system than in the conventional telepathology system in a certain limited time.

\section{Conclusion}

VS system was used for quick-frozen intra-operative telepathology diagnosis in Kyoto, Japan. The VS system was found to attain correct diagnosis on time, i.e., VS making within $10 \mathrm{~min}$ and VS telepathology observations/diagnoses within the next $5 \mathrm{~min}$. The novel VS telepathology system was found to be superior to the conventional still-image system in terms of time required for diagnosis and of ease and accuracy of the diagnosis. The use of VS system will be expanded from education to actual diagnosis including quick frozen intra-operative diagnosis. In the near future the conventional telepathology system to support regional medicine will be replaced by VS telepathology system.

\section{Acknowledgements}

This article has been published as part of Diagnostic Pathology Volume 3 Supplement I, 2008: New trends in digital pathology: Proceedings of the 9th European Congress on Telepathology and 3rd International Congress on Virtual Microscopy. The full contents of the supplement are available online at http://www.diagnosticpathology.org/supplements/3/SI

\section{References}

I. Yasunari Tsuchihashi, Masahiro Morishima, Kanae Nakasato, Ikuo Tofukuji, Setsuya Fujita: Shift to the broadband in practical telepathology in Kyoto, Japan. Abstract. 8th European Congress on Telepathology and 2nd International Congress on Virtual Microscopy 2006:91.

2. Claro automatic virtual microscopy [http://www.claro-inc.jp/ global/index.html]

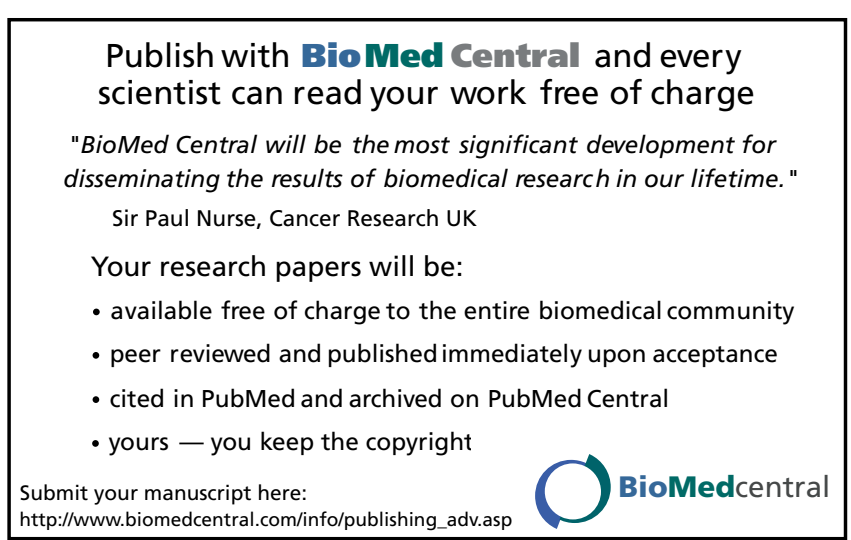

